\title{
Synthesis and Accumulation of Glutenin Subunits during Grain Development in Bread Wheat as Revealed by Reversed-phase Ultra-performance Liquid Chromatography (RP-UPLC)
}

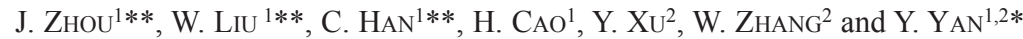 \\ ${ }^{1}$ College of Life Science, Capital Normal University, Beijing 100048, China \\ ${ }^{2}$ Hubei Collaborative Innovation Center for Grain Industry, 434025 Jingzhou, China
}

(Received 18 September 2015; Accepted 30 October 2015;

Communicated by F. Békés)

\begin{abstract}
Wheat glutenins containing high and low molecular weight glutenin subunits (HMW-GS and LMW-GS) are the major determinants of wheat gluten quality. In this study, the recently developed reversed-phase ultra-performance liquid chromatography (RP-UPLC) was used to study the synthesis and accumulation patterns of glutenins during grain development of four Chinese bread wheat cultivars with different gluten quality. Developing grains were collected based on thermal times from $150{ }^{\circ} \mathrm{Cd}$ to $750{ }^{\circ} \mathrm{Cd}$ at $100^{\circ} \mathrm{Cd}$ intervals, and the content of glutenin subunits and their accumulation patterns were determined by RP-UPLC as well as sodium dodecyl sulfate polyacrylamide gel electrophoresis (SDS-PAGE). The results showed that HMW-GS and LMW-GS synthesis were initiated currently at $250{ }^{\circ} \mathrm{Cd}$ and they displayed a gradually upregulated expression. All the HMW-GS can be detected at $250^{\circ} \mathrm{Cd}$, earlier than LMW-GS. Different glutenin subunits and genotypes showed clear accumulation diversity during grain development. Particularly, 1Dx5+1Dy10 in the cultivar Gaocheng 8901 and Zhongyou 9507 with superior dough properties were accumulated faster at early stages than 1Dx2+1Dy12 in Jingdong 8 and Zhengmai 9023 with poor dough quality, suggesting that faster accumulation rate of glutenin proteins at the early stages of grain development may contribute to the formation of superior gluten structure and dough quality.
\end{abstract}

Keywords: wheat, glutenins, accumulation, RP-UPLC

Abbreviations: ACN, acetonitrile; BiP, binding protein; DPA, days post anthesis; GC, Gaocheng 8901; HMW-GS, high molecular weight glutenin subunits; JD, Jingdong 8; LMW-GS, ligh molecular weight glutenin subunits; PDI, proteins disulfide isomerase; RP-HPLC, reversed-phase high-performance liquid chromatography; RP-UPLC, reversedphase ultra-performance liquid chromatography; SDS-PAGE, sodium dodecyl sulfate polyacrylamide gel electrophoresis; TFA, trifluoracetic acid; ZM, Zhengmai 9023; ZY, Zhongyou 9507

\footnotetext{
*Corresponding author; E-mail: yanym@cnu.edu.cn; Phone and Fax: +86-10-68902777

**Contributed equally to this work.
} 


\section{Introduction}

Wheat (Triticum aestivum L., $2 \mathrm{n}=6 \mathrm{x}=42$, AABBDD) is one of the most important cereal crops in the world. Wheat flour can be used to make various food products such as bread, noodle, cakes biscuits and pasta. The grain proteins of wheat are traditionally classified into two groups: non-storage protein and storage protein. The storage proteins consisting of polymeric glutenins and monomeric gliadins, account for $40 \%$ of all wheat grain proteins, which are the main determinant of the end-use characteristics of wheat flours (Weegels et al. 1996). The polymeric glutenins include high and low molecular weight glutenin subunits (HMW-GS and LMW-GS) with 70-140 kDa and 30-50 kDa, respectively, and impart viscoelasticity to dough while monomeric gliadins $(30-80 \mathrm{kDa})$ confer dough extensibility (Payne 1987).

HMW-GS are encoded by the Glu-1 loci (Glu-A1, Glu-B1, and Glu-D1) on the long arms of homoeologous group 1 chromosomes while the major LMW-GS are encoded by the complex Glu-3 locus on the short arms of chromosomes 1A, 1B and 1D (Payne 1987). The significant relationships between some HMW-GS and strong dough characteristics have been found so far (Payne 1987; Redaelli et al. 1997; Gianibelli et al. 2001). For instance, allelic pair 1Dx5 + 1Dy10 subunits have stronger dough quality than the allelic pair 1Dx2 + 1Dy12 and 1Dx4 + 1Dy12 (Payne 1987; Shewry et al. 2003). The gene copy numbers of LMW-GS have been estimated to vary from 10-15 to 35-40 in hexaploid wheat (Zhu and Khan 1999; Shewry et al. 2003). The allelic variations of LMW-GS are also closely related to different dough properties in wheat cultivars (Payne and Lawrence 1983; An et al. 2005).

To date, various methods have been developed to study the synthesis and accumulation patterns of wheat storage proteins, including the mostly used traditional sodium dodecyl sulfate polyacrylamide gel electrophoresis (SDS-PAGE) and reversed-phase (RP) and size-exclusion (SE) high-performance liquid chromatography (HPLC) (Gupta et al. 1993; Liu et al. 2012). Glutenins can be detected from as early as 7 days post anthesis (DPA) by using SE-HPLC (Gupta et al. 1993) and RP-HPLC (Liu et al. 2012). SDS-PAGE can detect glutenin synthesis at 7-14 DPA (Ng et al. 1990; Liu et al. 2012) depend on the materials used and their growth environments. Glutenins of the cultivars with 1Dx5+1Dy10 subunits were found to accumulate larger polymers more quickly than with 1Dx2+1Dy12 at early grain development stage (Gupta et al. 1993). However, these methodologies are generally time-consuming, sophisticated and imprecise.

Along with the development of protein separation methods, a more powerful technique reversed-phase ultraperformance liquid chromatography (RP-UPLC) based on traditional RP-HPLC has been developed (Wu et al. 2006). Compared with RP-HPLC, more rapid separation, higher resolution and sensitivity could be obtained by RP-UPLC analysis (Swartz 2005). Recently, RP-UPLC has received more and more attention and has been successfully applied to separate and characterize grain water-soluble proteins, glutenins and gliadins of wheat (Yu et al. 2013a, b; Zhou et al. 2013; Yan et al. 2014; Han et al. 2015). In the present work, we focused on the investigation for the synthesis and dynamic accumulation of HMW-GS and LMW-GS during grain development among four 
Chinese bread wheat cultivars with different gluten quality properties using the recently developed RP-UPLC method in our laboratory. Our results provide useful information for better understanding the expression profiles and functional properties of wheat glutenins.

\section{Materials and Methods}

\section{Wheat materials, field cultivation and sampling}

Four bread wheat cultivars (Triticum aestivum L., $2 \mathrm{n}=6 \mathrm{x}=42$, AABBDD) widely cultivated in China were used in this study: Gaocheng 8901 (GC) and Zhongyou 9507 (ZY) with 1Dx5 + 1Dy10 and superior dough properties, Jingdong 8 (JD) and Zhengmai 9023 (ZM) with 1Dx2 + 1Dy12 and poor dough properties (Chen et al. 2005). They were planted at the experimental station of the Chinese Academy of Agriculture Sciences (CAAS), Beijing during 2014-2015 growth season. Field experiments were performed in randomized block design with three biological replicates (each plot with $20 \mathrm{~m}^{2}$ ). The cultivation and management were same as local field cultivation conditions. The ears were tagged on the day of anthesis, and grain samples from middle ears were harvested at seven different grain developmental periods based on thermal times from $150{ }^{\circ} \mathrm{Cd}$ to $750{ }^{\circ} \mathrm{Cd}$ (fully ripened) at $100^{\circ} \mathrm{Cd}$ intervals, and then stored in liquid nitrogen prior to use.

\section{Protein extraction}

Grain samples were ground into fine powder using a mortar and glutenin proteins were extracted according to Liu et al. (2012). All samples were used for SDS-PAGE and RPUPLC analyses within $24 \mathrm{~h}$ of extraction.

\section{$S D S-P A G E$}

SDS-PAGE was carried out according to Yan et al. (2003). The gels were stained with 1\% Coomassie brilliant blue for $24 \mathrm{~h}$ and then destained with solution containing $10 \%$ ethanol and 10\% acetic acid. A GS-800 Calibrated Densitometer (Bio-Rad, Dallas, Texas, USA) was used to scan the gel.

\section{$R P-U P L C$}

RP-UPLC procedure for glutenin separation was performed based on Yan et al. (2014) and $\mathrm{Yu}$ et al. (2013b) on an Acquity UPLC (Waters Corp.) with a Waters 300SB C18 column $(1.7 \mu \mathrm{m})$. The mobile phases were $\mathrm{ddH}_{2} \mathrm{O}$ containing $0.1 \%$ trifluoracetic acid (TFA) and acetonitrile (ACN) with 1\% TFA, respectively, and UPLC grade solvents were used in all cases. Glutenins were dissolved in solvent $(21 \% \mathrm{ACN}, 0.1 \% \mathrm{TFA})$ and $10 \mu \mathrm{L}$ samples were injected and eluted with a linear gradient of $21 \%$ to $47 \%$ ACN (containing $0.1 \% \mathrm{TFA}$ ) at $0.2-0.6 \mathrm{~mL} / \mathrm{min}$ flow rates and elute times from 10 to $55 \mathrm{~min}$. The column 
temperature was set as $60^{\circ} \mathrm{C}$ and the protein peaks were detected by UV with absorbance areas at $210 \mathrm{~nm}$. The data of peak areas were used to generate the protein accumulation profile. The data reported are the average of three biological repeats.

\section{Results}

Glutenin synthesis and accumulation during grain development revealed by SDS-PAGE

The relative mobility orders from cathode to anode of HMW-GS separated by SDS-PAGE are: $1 \mathrm{Ax} \rightarrow 1 \mathrm{Dx} \rightarrow 1 \mathrm{Bx} \rightarrow 1 \mathrm{By} \rightarrow 1 \mathrm{Dy}$ based on the molecular weight (Payne and Lawrence, 1983; Yan et al., 2003). Firstly, the accumulation patterns of glutenins from four cultivars were studied by SDS-PAGE and the results were shown in Fig. S1*. The results showed that HMW-GS compositions of four bread wheat cultivars were: Zhongyou 9507 (1Ax1, 1Bx7+1By9, 1Dx5 + 1Dy10), Zhengmai 9023 (Null, 1Bx7+1By8, 1Dx2 + 1Dy12), Gaocheng 8901 (1Ax1, 1Bx7 + 1By8, 1Dx5 + 1Dy10) and Jingdong 8 (Null, 1Bx7 + 1By9, 1Dx2 + 1Dy12). Both HMW-GS and LMW-GS synthesis was initiated at $250{ }^{\circ} \mathrm{Cd}$ after anthesis and then gradually upregulated until grain maturity. At $250^{\circ} \mathrm{Cd}$, only trace amounts of Glu-A1 and Glu-B1 subunits, and clear Glu-D1 subunits

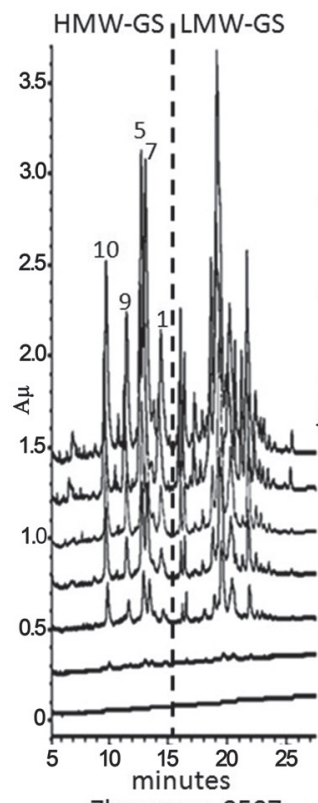

Zhongyou 9507

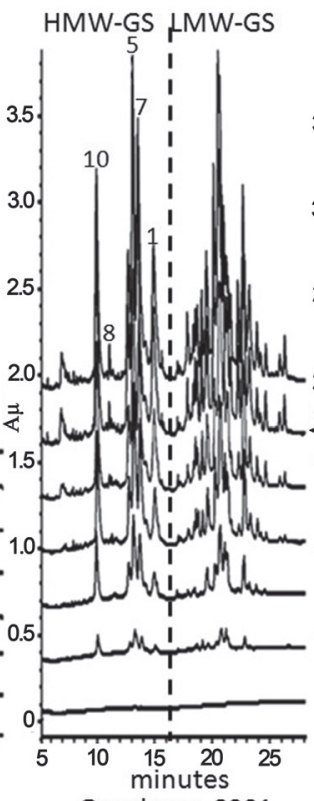

Gaocheng 8901

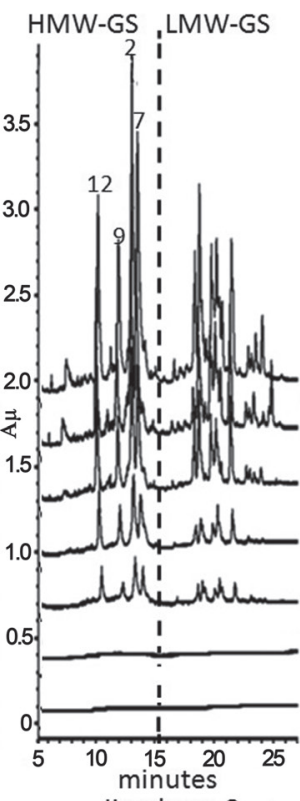

Jingdong 8

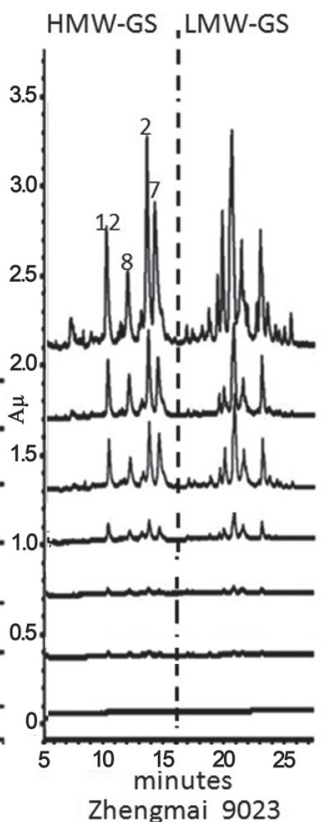

Zhengmai 9023

Figure 1. Accumulation patterns of glutenins at seven grain developmental stages from $150{ }^{\circ} \mathrm{Cd}$ to $750{ }^{\circ} \mathrm{Cd}$ in four Chinese bread wheat cultivars by RP-UPLC. The $x$ axis is the elution time (min) and the $y$ axis is the UV absorbance areas at $210 \mathrm{~nm}$. HMW-GS compositions were indicated

*Further details about the Electronic Supplementary Material (ESM) can be found at the end of the article. 
could be detected in GC, ZY, and ZM (lanes 1-7, 9-14 and 16-22). In JD, bands of HMW-GS could be faintly observed at $250^{\circ} \mathrm{Cd}$, but could be clearly detected after $350^{\circ} \mathrm{Cd}$ (lanes 24-30). Most of LMW-GS bands were initiated at $250^{\circ} \mathrm{Cd}$, and some of them could be detected after $450^{\circ} \mathrm{Cd}$. However, some LMW-GS bands could be observed after $650^{\circ} \mathrm{Cd}$. Two interesting LMW-GS bands marked with A, B in Fig. S1 in Jingdong 8 were detected, in which the band $\mathrm{A}$ was suddenly disappeared at $650^{\circ} \mathrm{Cd}$ while the band B only appeared at $650^{\circ} \mathrm{Cd}$.

\section{Synthesis and accumulation patterns of glutenin subunits revealed by RP-UPLC}

The recent optimized RP-UPLC procedure in our group (Yan et al. 2014) was used to separate and characterize glutenin subunits. As shown in Fig. 1, the relative migration order of HMW-GS by RP-UPLC based on their surface hydrophobicity is $1 \mathrm{Dy} \rightarrow 1 \mathrm{By} \rightarrow 1 \mathrm{Dx} \rightarrow 1 \mathrm{Bx} \rightarrow 1 \mathrm{Ax}$. Both HMW-GS and LMW-GS were synchronously synthesized at $250-350{ }^{\circ} \mathrm{Cd}$, they accumulated gradually and reached to the highest levels

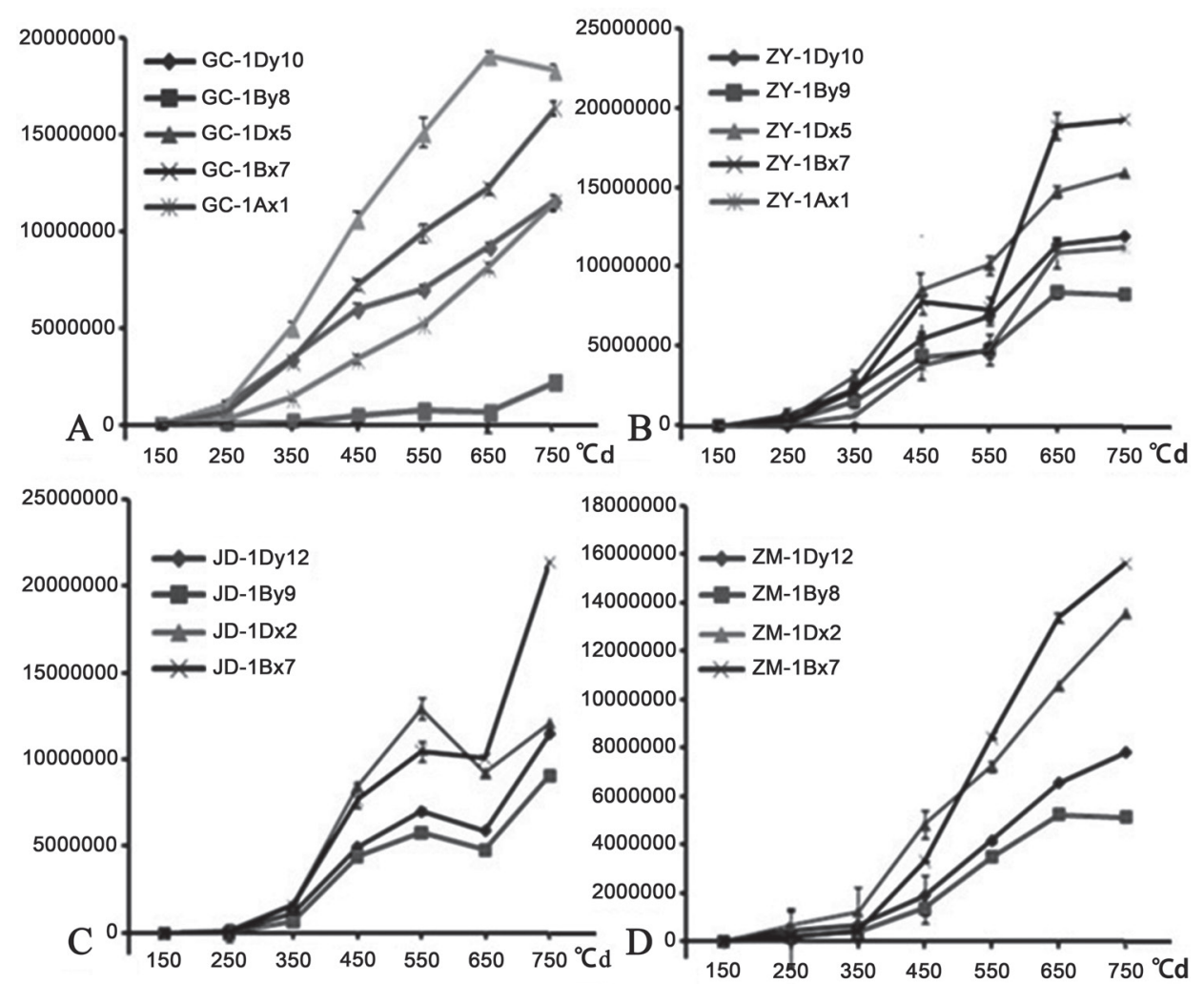

Figure 2. The accumulation patterns of individual HMW-GS in four cultivars during grain development stages. (A) GC - Gaocheng 8901; (B) ZY - Zhongyou 9507; (C) JD - Jingdong 8; (D) ZM - Zhengmai 9023. The $x$ axis is the grain developmental stages $\left({ }^{\circ} \mathrm{Cd}\right)$ and the $y$ axis is the $\mathrm{UV}$ absorbance areas at $210 \mathrm{~nm}$ 
at grain maturity afterward (Fig. 1). Also, no glutenin peaks were detected at $150{ }^{\circ} \mathrm{Cd}$, and most of HMW-GS could be identified at $250^{\circ} \mathrm{Cd}$ except for $1 \mathrm{By} 9$ in JD at $350^{\circ} \mathrm{Cd}$. At $250{ }^{\circ} \mathrm{Cd}$, LMW-GS could be clearly detected only in GC, and weekly detected in both ZY and $\mathrm{ZM}$, but could not be detected in JD, demonstrating a clear time difference of glutenin synthesis in different genotypes. After $350^{\circ} \mathrm{Cd}$, the amount of both HMW-GS and LMWGS generally showed a rapid increase until grain maturity, consistent with the results revealed by SDS-PAGE.

The accumulation patterns of individual HMW-GS in four cultivars were showed in Fig. 2A-D. During grain development, the expression levels of HMW-GS in the three cultivars showed very few changes until $250^{\circ} \mathrm{Cd}$, and then displayed a rapid accumulation until maturity. The subunit content order in the mature grains was $1 \mathrm{Bx}>$ glu$1 \mathrm{Dx}>1 \mathrm{Dy}>1 \mathrm{By}$ except for in GC, in which $1 \mathrm{Dx} 5$ was higher than $1 \mathrm{Bx} 7$. At the early stages, both 1Dx and 1Dy subunit contents were higher than 1B subunits in all four cultivars. Then $1 \mathrm{Dx} 5$ was exceed by $1 \mathrm{Bx} 7$ at $550{ }^{\circ} \mathrm{Cd}$ in $\mathrm{ZM}, 650^{\circ} \mathrm{Cd}$ in $\mathrm{ZY}$ and JD, but $1 \mathrm{By}$ subunits were always lower than 1Dy subunits. The 1Ax1 was always at the middle of $1 \mathrm{By} 8$ and $1 \mathrm{Dy} 10$ in $\mathrm{GC}$, and lower than $1 \mathrm{By} 9$ before $650{ }^{\circ} \mathrm{Cd}$ in $\mathrm{ZY}$. Interestingly, individual subunits shared similar accumulation patterns in each cultivars: gradual upregula-
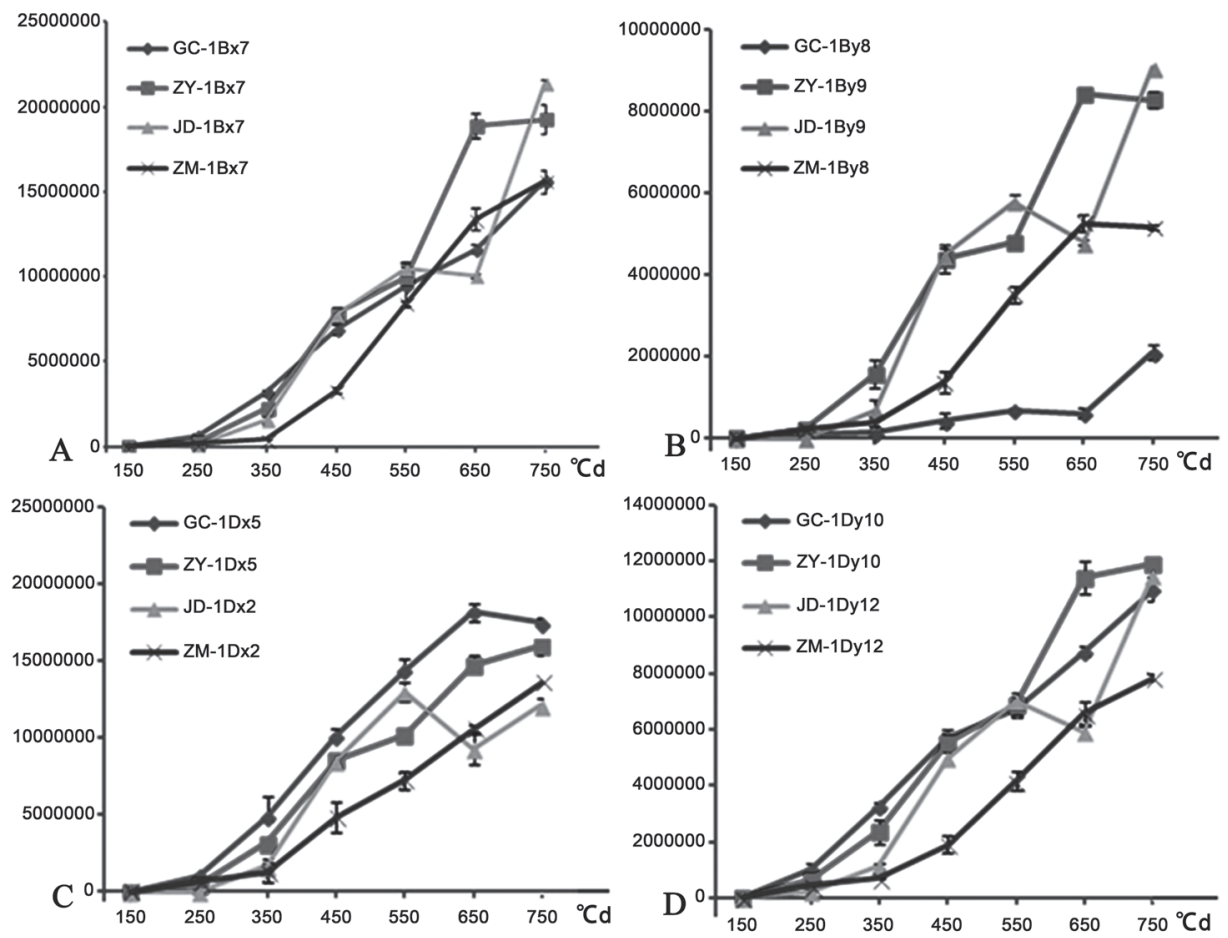

Figure 3. Comparison of the accumulation patterns of HMW-GS encoded by the same loci in four bread wheat cultivars during seven grain development stages. (A) 1Bx subunits; (B) 1By subunits; (C) 1Dx subunits; (D) 1Dy subunits. The $x$ axis is the grain developmental stages $\left({ }^{\circ} \mathrm{Cd}\right)$ and the $y$ axis is the $\mathrm{UV}$ absorbance areas at $210 \mathrm{~nm}$ 
tion from $150^{\circ} \mathrm{Cd}$ to $750^{\circ} \mathrm{Cd}$. Except for $1 \mathrm{Dx} 5 \mathrm{in} \mathrm{GC}$ with a little decreasing at $650^{\circ} \mathrm{Cd}$, all subunits in GC (Fig. 2A) and ZM (Fig. 2D) were increased linearly from $350^{\circ} \mathrm{Cd}$ to grain maturity. Particularly, HMW-GS had a plateau at $4500^{\circ} \mathrm{Cd}$ and $6500^{\circ} \mathrm{Cd}$ in $\mathrm{ZY}$ (Fig. 2B), but with a little decreasing at $650^{\circ} \mathrm{Cd}$ in JD (Fig. 2C).

\section{Comparison of glutenin accumulation patterns between different cultivars}

The comparison of accumulation patterns of HMW-GS at the same loci between different cultivars were showed in Fig. 3. The kinetic accumulation of $1 \mathrm{Bx}$ subunits in GC and ZY was increased faster at early stages than that in JD and ZM. But $1 \mathrm{Bx} 7+1 \mathrm{By} 9$ subunits in $\mathrm{JD}$ had a remarkable increase from $650^{\circ} \mathrm{Cd}$ and $750^{\circ} \mathrm{Cd}$ (Fig. 3A-B). The $1 \mathrm{By} 8$ subunit in $\mathrm{GC}$ was slowly increased from $150^{\circ} \mathrm{Cd}$ and $650{ }^{\circ} \mathrm{Cd}$, but significant upregulation from $650^{\circ} \mathrm{Cd}$ and $750^{\circ} \mathrm{Cd}$, which showed a much lower expression level than other $1 \mathrm{By}$ subunits during all grain developmental stages (Fig. 3B). The 1D subunits in GC and ZY always had higher expression levels than those in JD and ZM except for 1Dx2 in JD with a higher content at $550^{\circ} \mathrm{Cd}$ (Fig. 3C-D).
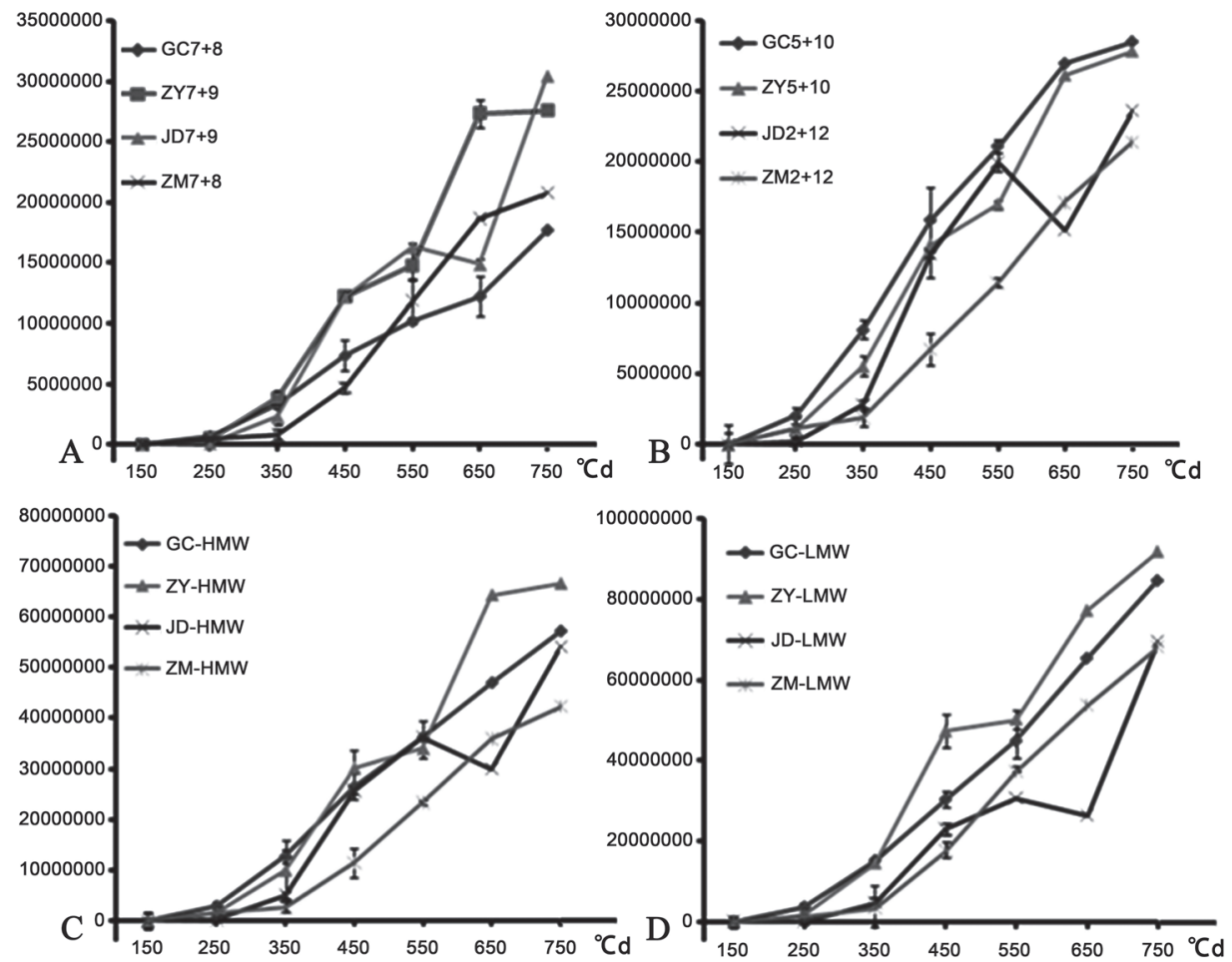

Figure 4. Comparison of the accumulation patterns of different glutenin groups in four bread wheat cultivars during grain development stages. (A) 1B subunits; (B) 1D subunits; (C) Total HMW-GS; (D) Total LMW-GS.

The $x$ axis is the grain developmental stages $\left({ }^{\circ} \mathrm{Cd}\right)$ and the $y$ axis is the $\mathrm{UV}$ absorbance areas at $210 \mathrm{~nm}$ 
The accumulation curves of HMW-GS encoded by Glu-1D, Glu-1B, total HMW-GS content, and total LMW-GS content in four bread wheat cultivars during different grain developmental stages were shown in Fig. 4A-D. The total amounts of HMW-GS, LMWGS, 1Dx $5+1 \mathrm{Dy} 10 / 1 \mathrm{Dx} 2+1 \mathrm{Dy} 12$ and $1 \mathrm{Bx} 7+1 \mathrm{By} 8 / 1 \mathrm{Bx} 7+1 \mathrm{By} 9$ in each cultivar shared similar increasing accumulation patterns with individual subunits. The content of glutenins at Glu-1B, Glu-1D, total HMW-GS content, and total LMW-GS content were also accumulated faster in GC and ZY than those in $\mathrm{ZM}$ and JD before $450{ }^{\circ} \mathrm{Cd}$ like individual subunits. Particularly, clear accumulation differences between $1 \mathrm{Dx} 5+1 \mathrm{Dy} 10$ and $1 \mathrm{Dx} 2+1 \mathrm{Dy} 12,1 \mathrm{Bx} 7+1 \mathrm{By} 8$ and $1 \mathrm{Bx} 7+1 \mathrm{By} 9$ were found. In GC and ZY, 1Dx5 + 1Dy 10 subunits were rapidly accumulated in the beginning of $150^{\circ} \mathrm{Cd}$, however, $1 \mathrm{Dx} 2+1 \mathrm{Dy} 12$ subunits in JD and ZM increased slowly until $350^{\circ} \mathrm{Cd}$. Similarly, $1 \mathrm{Bx} 7+1 \mathrm{By} 9$ in ZY and JD had an higher accumulation rate during grain development and a higher protein content in the mature grains than those of $1 \mathrm{Bx} 7+1 \mathrm{By} 8$ in GC and ZM (Fig. 4A-B).

\section{Discussion}

Bread wheat is an allohexaploid species with a huge genome (up to $17 \mathrm{~Gb}$ ). Seed storage proteins are encoded by a multigene family in which extensive allelic variations are present. Thus, the expression and regulation of glutenins appear to be complex. Previous reports demonstrated some differences for the synthesis and accumulation of storage proteins, possibly resulted from different materials used and their growth environments. HMW-GS, LMW-GS and gliadins occurred concurrently at as early as 7 DPA (Gupta et al. 1996; Liu et al. 2012). But in the report of Panozzo et al. (2001), LMW-GS was synthesized later than HMW-GS and negligible concentrations could be detected before 14 DPA. Dutta et al. (2011) found that the temporal synthesis of different gliadin and glutenin proteins was cultivar dependent. Particularly, 1Dx5 + 1Dy10 subunits were found to accumulate more quickly than $1 \mathrm{Dx} 2+1 \mathrm{Dy} 12$ at early stages of grain development (Gupta et al. 1996). In the current study, HMW-GS and LMW-GS were observed concurrently at $250{ }^{\circ} \mathrm{Cd}$ after anthesis when detected by RP-UPLC (Fig. 1). All HMW-GS can be observed at $350^{\circ} \mathrm{Cd}$ whereas complete LMW-GS can be detected at $550{ }^{\circ} \mathrm{Cd}$, later than HMW-GS. Different accumulated patterns were present in different glutenin subunits cultivars. 1Dx5+1Dy10 were also found to have higher accumulation rate at the early stages of grain development than 1Dx2+1Dy12 (Fig. 4B), which may contribute to the formation of superior gluten structure and dough quality.

Wheat grains contain monomeric gliadins and polymeric glutenins that form gluten macropolymer through inter- and intra-molecular bisulfide bonds and determine the processing qualities of a wide range of end products (Wrigley 1996). The quantity and size distribution of polymeric protein have important effects on flour quality properties (Gupta et al. 1993). Studies showed that significant changes in size distribution of the polymers occurred during the late grain developmental stages, consistent with a rapid increase in the amounts of the glutenin subunits as shown in this work and previous report (Gupta et al. 1996). In particular, the wheat cultivars with 1 Dx $5+1$ Dy 10 showed to accumulate larger polymers more quickly than those with allelic subunits $1 \mathrm{Dx} 2+1 \mathrm{Dy} 12$ 
(Gupta et al. 1996). Thus, the rapid accumulation of 1Dx5 + 1Dy10 could benefit larger polymer formation, and then improve gluten quality.

The glutenins are typical secretory proteins synthesized in polyribosomes and attached to rough endoplasmic reticulum (ER) in where disulfide bond formation, glutenin protein folding and maturation occur under the help of ER lumenal proteins such as molecular chaperone proteins disulfide isomerase (PDI) and binding protein (BiP) (Bollini and Chrispeels 1979; Shewry 1999; Tosi et al. 2009). PDI gene family containing thioredoxin domains is involved in the correct formation of disulphide bond, protein folding and assembling of protein bodies. The endoplasmic reticulum chaperone BiPs also participate in protein synthesis, folding assembly, and secretion. Recent studies have indicated that PDIL1-1 and PDIL12-1 have the highest expression level at the early grain developmental stage at 10 DPA, PDIL4-1 and PDIL5-1 were also highly active at 5 DPA (Wang et al. 2013; Zhu et al. 2014a). Hurkman et al. (2009) also found that PDI had very abundant content at early stages of grain development. More recently, Zhu et al. (2014b) has demonstrated that TaBip has the highest expression level at 14 DPA. The high expression of those molecular chaperones along with the rapid accumulation rate of 1Dx $5+1$ Dy 10 subunits at early grain development stages could facilitate the folding of gluten proteins and help to bring about the formation of more regular gluten polymers, which might result in superior gluten quality. This may be a reason that the cultivars Zhongyou 9507 and Gaocheng 8901 with 1Dx5 + 1Dy10 have better bread making quality than Zhongmai 175 and Jingdong 8 with 1Dx2 + 1Dy12.

RP-UPLC is capable of the precise quantitative analysis as well as faster speed, higher resolution, more sensitivity and small reagent and sample requirement, providing a more powerful tool for the studies on the synthesis and accumulation of seed storage proteins. The widely used conventional SDS-PAGE and RP-HPLC methods for glutenin separation have clear disadvantages such as time-consuming and labor-intensive procedures with relatively poor resolution. Thus, RP-UPLC can overcome these drawbacks and has greater application potential for the studies on the synthesis and accumulation mechanisms of seed storage proteins as well as the rapid screening for the desirable glutenin subunits in the early generations of wheat quality improvement.

\section{Acknowledgements}

This research was financially supported by grants from the National Natural Science Foundation of China (31471485), Natural Science Foundation of Beijing City and the Key Developmental Project of Science Technology, Beijing Municipal Commission of Education (KZ201410028031).

\section{References}

An, X., Li, Q., Yan, Y., Xiao, Y., Hsam, S.L.K., Zeller, F.J. 2005. Genetic diversity of European spelt wheat (T. aestivum ssp. spelta L. em. Thell.) revealed by glutenin subunit variations at the Glu-1 and Glu-3 loci. Euphytica 146:193-201. 
Bollini, R., Chrispeels, M.J. 1979. The rough endoplasmic reticulum is the site of reserve-protein synthesis in developing Phaseolus vulgaris cotyledons. Planta 146:487-501.

Chen, D.L., Zhang, Y., He, Z.H., Wang, D.S., Peña-Bautista, R.J. 2005. Effect of water addition on northern style Chinese steamed bread processing quality. Acta Agron. Sin. 6:730-735.

Dutta, T., Kaur, H., Singh, S., Mishrac, A., Tripathic, J.K., Singhb, N., Pareekd, A., Singha, P. 2011. Developmental changes in storage proteins and peptidyl prolyl cis-trans isomerase activity in grains of different wheat cultivars, Food Chem. 128:450-457.

Gianibelli, M., Larroque, O., MacRitchie, F., Wrigley, C.W. 2001. Biochemical, genetic, and molecular characterization of wheat glutenin and its component subunits. Cereal Chem. 78:635-646.

Gupta, R.B., Khan, K., MacRitchie, F. 1993. Biochemical basis of flour properties in breadwheats. I: Effects of variation in the quantity and size distribution of polymeric protein. J. Cereal Sci. 18:23-41.

Gupta, R.B., Masci, S., Lafiandra, D., Bariana, H.S., MacRitchie, F. 1996 Accumulation of protein subunits and their polymers in developing grains of hexaploid wheats. J. Exp. Bot. 47:1377-1385.

Han, C., Lu, X., Yu, Z., Li, X., Ma, W., Yan, Y. 2015. Rapid separation of seed gliadins by reversed-phase ultraperformance liquid chromatography (RP-UPLC) and its application in wheat variety and germplasm identification. Biosci. Biotechnol. Biochem. 79:808-815.

Hurkman, W.J., Vensel, W.H., Tanaka, C.K., Whitehand, L., Altenbach, S.B. 2009. Effect of high temperature on albumin and globulin accumulation in the endosperm proteome of the developing wheat grain. J. Cereal Sci. 49:12-23.

Liu, W., Zhang, Y., Gao, X., Wang, K., Wang, S., Zhang, Y., He, Z., Ma, W., Yan, Y. 2012. Comparative proteome analysis of glutenin synthesis and accumulation in developing grains between superior and poor quality bread wheat cultivars. J. Sci. Food Agric. 92:106-115.

Ng, P.K.W., Slominski, E., Johnson, W.J., Bushuk, W. 1990. Changes in wheat endosperm proteins during grain maturation. In: Bushuk, W., Tkachuk, R. (eds), Gluten Proteins 1990. American Association of Cereal Chemists (AACC). St. Paul, MN, USA. pp. 740-754.

Panozzo, J., Eagles, H.A., Wootton, M. 2001. Changes in protein composition during grain development in wheat. Aust. J. Agri. Res. 52:485-493.

Payne, P.I., Lawrence, G.J. 1983. Catalogue of alleles for the complex gene loci, Glu-A1, Glu-B1, and Glu-D1 which code for high-molecular-weight subunits of glutenin in hexaploid wheat. Cereal Res. Comm. 11:2935.

Payne, P. 1987. Genetics of wheat storage proteins and the effect of allelic variation on bread-making quality. Annu. Rev. Plant Physiol. 38:141-153.

Redaelli, R., Ng, P.K.W., Pogna, N.E. 1997. Allelic variation at the storage protein loci of 55 US-grown white wheats. Plant Breed. 116:429-436.

Shewry, P.R. 1999. The synthesis, processing and deposition of gluten proteins in the developing wheat grain. Cereal Foods World 44:587-589.

Shewry, P.R., Popineau, Y., Lafiandra, D., Halford, N.G., Tatham, A.S., Belton, P.S. 2003. The high molecular weight subunits of wheat glutenin and their role in determining wheat processing properties. Advances in Food Nutr. Sci. 45:219-302.

Swartz, M.E. 2005. UPLC: An introduction and review. J. Liquid Chromatogr. Relat. Technol. 28:1253-1263.

Tosi, P., Parker, M., Gritsch, C.S., Carzaniga, R., Martin, B., Shewry, P.R. 2009. Trafficking of storage proteins in developing grain of wheat. J. Exp. Bot. 60:979-991.

Wang, S., Yu, Z., Cao, M., Shen, X., Li, N., Li, X., Ma, W., Weißgerber, H., Zeller, F., Hsam, S., Yan, Y. 2013. Molecular mechanisms of HMW glutenin subunits from $1 \mathrm{~S}^{1}$ genome of Aegilops longissimi positively affecting wheat bread making quality. PLoS One 8:58947.

Weegels, P.L., Hamer, R.J., Schofield, J.D. 1996. Functional properties of wheat glutenin. J. Cereal Sci. 23:118.

Wrigley, C.W. 1996. Biopolymers-giant proteins with flour power. Nature 381:738-739.

Wu, Y., John, R., William, E., Hobbins, W.B. 2006. Ultra performance liquid chromatography (UPLC) further improves hydrogen/deuterium exchange mass spectrometry. J. Amer. Soc. Mass Spectrom. 17:163-167. 
Yan, X., Liu, W., Yu, Z.T., Han, C.X., Zeller, F.J., Hsam, S.L.K., Yan, Y.M. 2014. Rapid separation and identification of wheat HMW glutenin subunits by UPLC and comparative analysis with HPLC. Aust. J. Crop. Sci. 8:140-147.

Yan, Y., Hsam, S.L.K., Yu, J., Jiang, Y., Ohtsuka, I., Zeller, F.J. 2003. HMW and LMW glutenin alleles among putative tetraploid and hexaploid T. spelta progenitors. Theor. Appl. Genet. 107:1321-1330.

Yu, Z.T., Han, C.X., Wang, S., Lv, D.W., Chen, G.X., Li, X.H., Jiang, G.L., Yan, Y.M. 2013a. Fast separation and characterization of water-soluble proteins in wheat grains by reversed-phase ultra-performance liquid chromatography (RP-UPLC). J. Cereal Sci. 57:288-294.

Yu, Z., Han, C., Yan, X., Li, X., Jiang, G., Yan, Y. 2013b. Rapid characterization of wheat low molecular weight glutenin subunits by ultra-performance liquid chromatography (UPLC). J. Agric. Food Chem. 61:40264034.

Zhou, J.W., Han, C.X., Cao, H., Zhen, S.M., Yu, Z.T., Li, X.H., Ma, W.J., Yan, Y.M. 2013. Fast identification of wheat 1BL.1RS translocation by reversed-phase ultra-performance liquid chromatography (RP-UPLC). Crop Pasture Sci. 64:865-873.

Zhu, J., Khan, K. 1999. Characterization of monomeric and glutenin polymeric proteins of hard red spring wheats during grain development by multistacking SDS-PAGE and capillary zone electrophoresis. Cereal Chem. 76:261-269.

Zhu, C., Luo, N., He, M., Chen, G., Zhu, J., Yin, G., Li, X., Hu, Y., Li, J., Yan, Y. 2014a. Molecular characterization and expression profiling of the protein disulfide isomerase gene family in Brachypodium distachyon L.. PLoS One 9:e94704.

Zhu, J., Hao, P., Chen, G., Han, C., Li, X., Zeller, F.J., Hsam, S., Hu, Y., Yan, Y. 2014b. Molecular cloning, phylogenetic analysis, and expression profiling of endoplasmic reticulum molecular chaperone BiP genes from bread wheat (Triticum aestivum L.). BMC Plant Boil. 14:260.

\section{Electronic Supplementary Material (ESM)}

Electronic Supplementary Material (ESM) associated with this article can be found at the website of CRC at http://www.akademiai.com/content/120427/

Electronic Supplementary Figure S1. Synthesis and accumulation of glutenins revealed by SDS-PAGE. 1-7: Zhongyou 9507, $150-750{ }^{\circ} \mathrm{Cd}, 9-15$ : Zhengmai 9023, $150-750{ }^{\circ} \mathrm{Cd}, 16-22$ : Gaocheng $8901,150-750{ }^{\circ} \mathrm{Cd}$; 24-30: Jingdong 8, $150-750{ }^{\circ} \mathrm{Cd}, 8,23,31$ : Chinese Spring (CS). Band A suddenly disappeared at $650{ }^{\circ} \mathrm{Cd}$, Band $\mathrm{B}$ only appeared at $650^{\circ} \mathrm{Cd}$ 